\title{
Root-Finding by Fitting Rational Functions
}

\author{
By F. M. Larkin
}

\begin{abstract}
A tabular, recursive method is presented for the computation of a sequence of abscissae designed to converge to a simple zero of an analytic function. The key to the method is an efficient means for evaluating the zeros of a sequence of rational functions, having linear numerators, fitted to information previously computed.

Regional and asymptotic convergence properties of the method are described. Conditions sufficient to ensure convergence are derived, and it is shown that asymptotically quadratic convergence can be achieved, at the cost of only a moderate amount of "overhead" computation.
\end{abstract}

1. Introduction. A number of useful techniques (in particular the Secant Rule) for numerical determination of a zero $z_{0}$ of a function $f(\cdot)$ begin by sampling $f(\cdot)$ at distinct abscissae $\left\{z_{j} ; j=1,2, \ldots, r\right\}$, say, and fitting a "simpler" function $\widetilde{f}(\cdot)$ through the points $\left\{\left(z_{j}, f\left(z_{j}\right)\right) ; j=1,2, \ldots, r\right\}$. This construction is arranged so that a zero $z_{r+1}$ of $\widetilde{f}(\cdot)$ is relatively easy to compute. A new set of abscissae, including $z_{r+1}$, is then selected from $\left\{z_{j} ; j=1,2, \ldots, r+1\right\}$ and the process is iterated to generate a sequence of abscissae which, in favorable circumstances, will converge to $z_{0}$.

Tornheim [13] considered the choice of $\tilde{f}(\cdot)$ as a rational function, i.e.

$$
\tilde{f}(z)=\frac{P_{n}(z)}{Q_{m}(z)} ; \quad \forall z,
$$

where $P_{n}(\cdot)$ and $Q_{m}(\cdot)$ are polynomials of degree $n$ and $m$ respectively, such that $n+m+1=r$. Thus, $\tilde{f}(\cdot)$ has just the right number of degrees of freedom to be determined by the $r$ data points. In the same paper Tornheim also considered rational inverse interpolation, and obtained asymptotic convergence orders for both the direct and inverse case. Jarratt and Nudds [2] particularized to the case $n=1$, since then the determination of $z_{r+1}$ becomes trivial once $\widetilde{f}(\cdot)$ has been constructed. They confirm Tornheim's results on the asymptotic order of convergence in this case. However, Jarratt [4] later remarked that, for practical purposes, it is usually not worthwhile to choose anything other than $m=1$ (or, presumably, 0 ) when $n=1$, because of the relative labor of constructing $\tilde{f}(\cdot)$ by solving a set of $r$ simultaneous, linear equations for the coefficients of $P_{1}\left({ }^{\circ}\right)$ and $Q_{r-2}(\cdot)$.

The main purpose of this paper is to present a tabular, recursive technique for the determination of $z_{r+1}$, when $n=1$, without the necessity for computing the coefficients in $Q_{r-2}\left({ }^{\circ}\right)$. In order to augment the list $\left\{z_{j} ; j=1,2, \ldots, r\right\}$ by $z_{r+1}$

Received April 4, 1979.

1980 Mathematics Subject Classification. Primary $65 \mathrm{H} 05$. 
only $O(r)$ arithmetic operations are required, compared with $O\left(r^{3}\right)$ if the coefficients in $P_{1}(\cdot)$ and $Q_{r-2}\left({ }^{\circ}\right)$ are found by straightforwardly solving $r$ simultaneous, linear equations. Thus the computational work involved is comparable with that of the classical method of polynomial inverse interpolation, performed by the Neville-Aitken technique ([10], [1]).

The approach described here is also flexible to the extent that, if desired, earlier numbers of the sequence $\left\{z_{j} ; j=1,2,3, \ldots\right\}$ can be discarded at any stage, with no consequent computational penalty. Furthermore, by permitting certain abscissae to become confluent, it is a simple matter to include information on derivatives of $f(\cdot)$ within the general computational scheme. As a matter of interest, the technique can also be used for accelerating the convergence rate of abscissa sequences generated by a simple iterated mapping, by the Secant Rule, or even Newton's method, should that be considered worthwhile in order at any stage to make efficient use of all the previously computed information (Larkin [8]).

The conceptual basis of the method is discussed in Section 2, below, and the actual mechanics in Section 3. In Section 4 some sufficient convergence conditions are given and it is also shown that the asymptotic convergence order can be as high as 2. Since only a single function evaluation is required per step (in the nonconfluent case) this means that the efficiency index (Ostrowski [11]) and informational efficiency (Traub [14]) can also approach the value 2 . The asymptotic convergence rate is also governed by the proximity to the zero being sought of the nearest competitive zero or nonpolar singularity.

In view of the above properties, it seems that a root-finding method based on fitting the form

$$
f(z)=\frac{z-z_{r+1}}{Q_{r-2}(z)}
$$

to $r$ distinct sample points of $f(\cdot)$, and iterating with or without incrementing $r$ at each stage, may still deserve consideration as a possible practical technique.

2. The Table of Rational Interpolants. Fundamental to the approach is the table of rational interpolants illustrated in Figure 1, and the recognition that its members satisfy certain, simple recurrence relations. However, we emphasize that it will not be necessary actually to construct the interpolants per se.

In Figure 1 we define

$$
f_{j}=f\left(z_{j}\right) ; \quad j=1,2,3, \ldots, r
$$

and take

$$
R_{j 1}(z)=\frac{\left(z-z_{j}\right) f_{j+1}+\left(z_{j+1}-z\right) f_{j}}{z_{j+1}-z_{j}}, \quad \forall z ; j=1,2, \ldots, r-1 .
$$

For $k \geqslant 2$ we then take

$$
R_{j k}(z)=\frac{z-w_{j k}}{Q_{j k}(z)} ; \quad \forall z \text { and relevant } j, k
$$




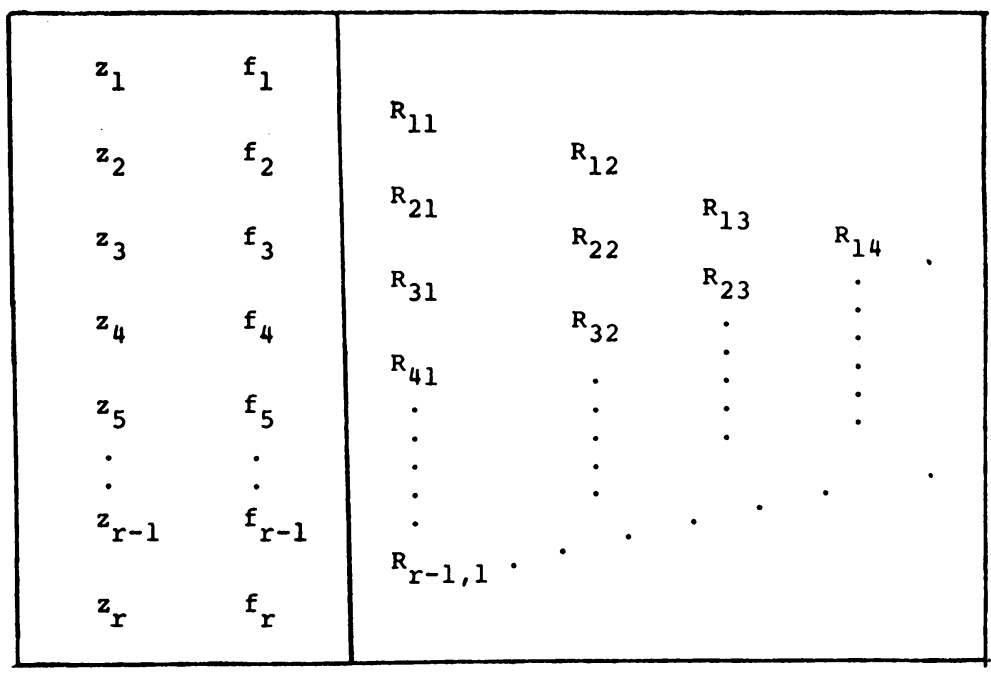

FiguRE 1

A table of rational interpolants

where $Q_{j k}(\cdot)$ is a polynomial of degree $\leqslant k-1$ whose $k$ coefficients, together with $w_{j k}$, are chosen so that

$$
R_{j k}\left(z_{s}\right)=f_{s} ; \quad s=j, j+1, j+2, \ldots, j+k
$$

presuming that to be possible. In the interests of clarity of presentation, we neglect the possibility that such a rational interpolant may not exist (nonexistence would not change the algorithm-merely its interpretation). Figure 2 illustrates the relationship in the table between $R_{j, k-1}, R_{j+1, k-1}$ and $R_{j k}$, and their defining data points.

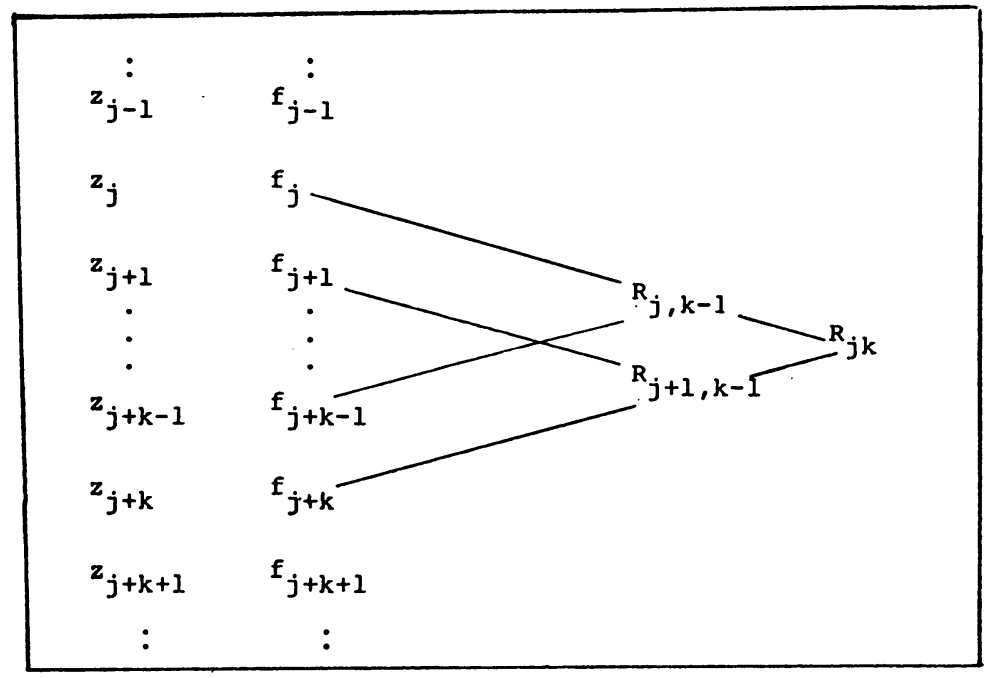

Figure 2

$R_{j k}$ and its neighbors 
Wynn [15], [16], Stoer [12] and Larkin [5] have all discussed different techniques for the recursive construction of rational interpolants. In this case the simplest approach seems to be as follows. Define a function $T(\cdot)$ by the relation

$$
T(z)=\frac{\left(z-z_{j}\right) \cdot\left(z-w_{j+1, k-1}\right)+\left(z_{j+k}-z\right) \cdot\left(z-w_{j, k-1}\right)}{\left(z-z_{j}\right) \cdot Q_{j+1, k-1}(z)+\left(z_{j+k}-z\right) \cdot Q_{j, k-1}(z)}, \quad \forall z,
$$

and observe that, presuming no cancellation of a linear function of the argument occurs, $T(\cdot)$ is a rational function whose numerator has degree 1 and whose denominator has degree $\leqslant k-1$. Furthermore,

$$
\begin{gathered}
T\left(z_{j}\right)=\frac{z_{j}-w_{j, k-1}}{Q_{j, k-1}\left(z_{j}\right)}=R_{j, k-1}\left(z_{j}\right)=f_{j}, \\
T\left(z_{j+k}\right)=\frac{z_{j+k}-w_{j+1, k-1}}{Q_{j+1, k-1}\left(z_{j+k}\right)}=R_{j+1, k-1}\left(z_{j+k}\right)=f_{j+k},
\end{gathered}
$$

and, because

$$
R_{j, k-1}\left(z_{s}\right)=f_{s}=R_{j+1, k-1}\left(z_{s}\right) ; \quad s=j+1, j+2, \ldots, j+k-1,
$$

we see that

$$
T\left(z_{s}\right)=f_{s} ; \quad s=j+1, j+2, \ldots, j+k-1 .
$$

Hence, by the uniqueness of rational interpolants,

$$
T(z)=R_{j k}(z), \quad \forall z,
$$

i.e.

(3) $R_{j k}(z)=\frac{z\left(w_{j, k-1}-z_{j}-w_{j+1, k-1}+z_{j+k}\right)-\left(z_{j+k} w_{j, k-1}-z_{j} w_{j+k, k-1}\right)}{\left(z-z_{j}\right) Q_{j+1, k-1}(z)+\left(z_{j+k}-z\right) Q_{j, k-1}(z)}, \quad \forall z$.

3. The Computational Scheme. Comparing (3) with (2), we see that

$$
w_{j k}=\frac{z_{j+k} w_{j, k-1}-z_{j} w_{j+k, k-1}}{w_{j, k-1}-z_{j}-w_{j+1, k-1}+z_{j+k}},
$$

or

$$
w_{j k}=\frac{w_{j+1, k-1} /\left(w_{j+1, k-1}-z_{j+k}\right)-w_{j, k-1} /\left(w_{j, k-1}-z_{j}\right)}{1 /\left(w_{j+1, k-1}-z_{j+k}\right)-1 /\left(w_{j, k-1}-z_{j}\right)}
$$

or

(6). $\quad w_{j k}=w_{j+1, k-1}+\frac{w_{j+1, k-1}-w_{j, k-1}}{\left(w_{j, k-1}-z_{j}\right) /\left(w_{j+1, k-1}-z_{j+k}\right)-1} ; \quad \forall j \geqslant 1 ; k \geqslant 2$.

Any of the algebraically equivalent relations (4), (5) and (6) constitute a recurrence rule, by means of which the quantities $\left\{w_{j, k} ; j=1,2,3, \ldots\right\}$ can be constructed if the values of the $\left\{w_{j, k-1} ; j=1,2,3, \ldots\right\}$ are known. Of course, the values of the $\left\{w_{j 1} ; j=1,2,3, \ldots\right\}$ are nothing other than Secant Rule estimates of $z_{0}$, 
given by

$$
w_{j 1}=z_{j+1}-\left[\frac{z_{j+1}-z_{j}}{f_{j+1}-f_{j}}\right] f_{j+1} ; \quad j=1,2, \ldots, r-1 .
$$

Perhaps (6) is computationally the most convenient recurrence relation, since quite large relative errors in the increment part may be tolerated, without resulting in unduly large absolute errors in $w_{j k}$. A convenient layout for the quantities $\left\{w_{j k}\right\}$ is shown in Figure 3, the arrows indicating the direction of information flow during the course of the calculation. Figure 4 illustrates the order of calculation of the entries in this root-estimate table, starting with two initial estimates $z_{1}$ and $z_{2}$, and augmenting the list $\left\{z_{j} ; j=1,2,3, \ldots\right\}$ at each stage by means of the rule

$$
z_{r+1}=w_{1, r-1} ; \quad r=2,3,4, \ldots
$$

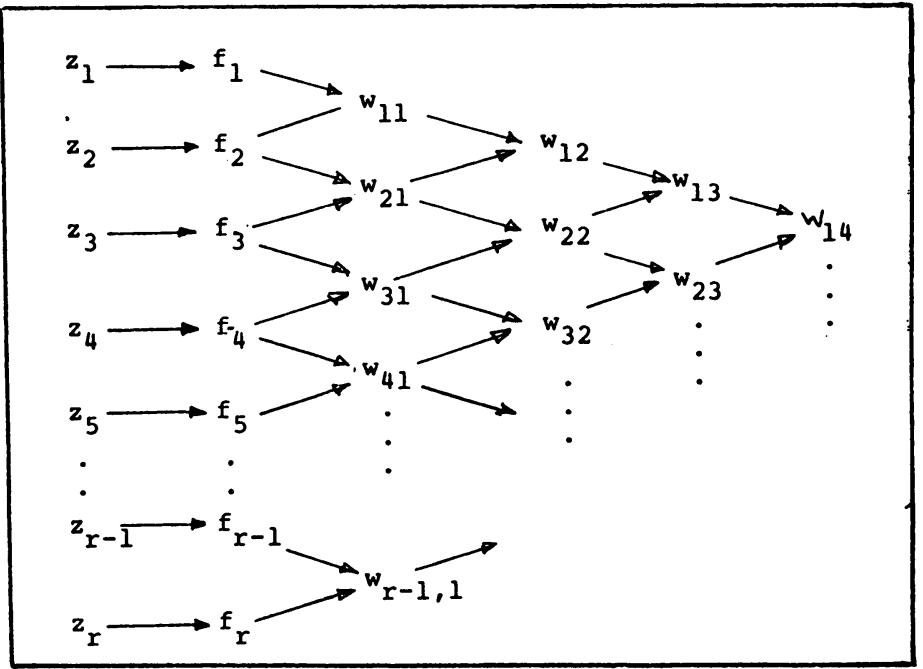

FIGURE 3

Information flow in the table of root estimates

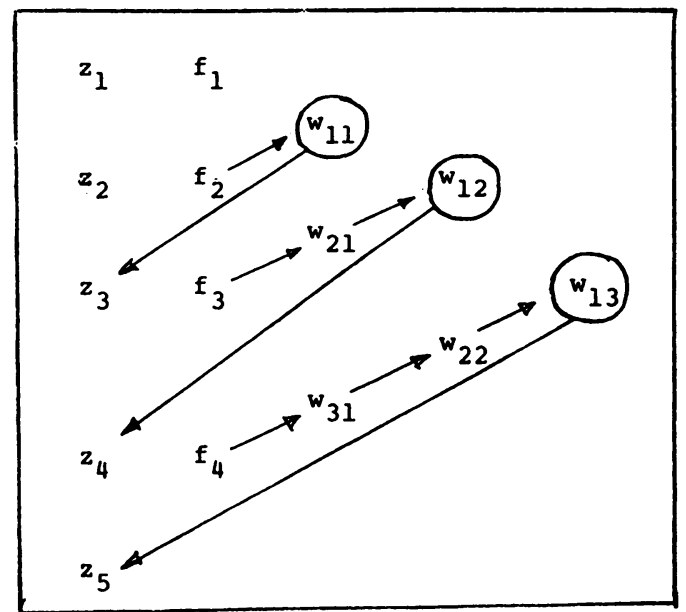

FIGURE 4

Order of calculation of root estimates 
Of course, many rules other than (7) could be used for augmenting the list, if desired. For example, we could take

$$
z_{r+1}=w_{r-2,2} ; \quad r=3,4,5, \ldots,
$$

in which case only the latest 4 list members are retained at each stage. It would even be possible to choose $z_{r+1}$ quite unrelated to previously computed entries in the table, for example, if $w_{1, r-1}$ turned out to be an obviously poor estimate of $z_{0}$.

Figure 5 illustrates the progress of the iteration, using augmentation rule (7), for the sample function

$$
f(z)=z-e^{-z} ; \quad \forall z .
$$

Correct digits are underlined. Starting from the same initial values, the simple Secant Rule requires six function evaluations in order to attain comparable accuracy, and starting from either 0 or 1 , the simple Newton-Raphson iteration requires four function and four derivative evaluations. Thus, while the improvement in efficiency is not spectacular, it would appear to be worthwhile for functions $f(\cdot)$ of only moderate complexity. The question of numerical stability of the method is currently being investigated; however, numerical experience suggests that it should not constitute a serious difficulty.

\begin{tabular}{|l|ll|lll|}
\hline$j$ & \multicolumn{1}{|c|}{$z_{j}$} & $f_{j}$ & $w_{j 1}$ & $w_{j 2}$ & $w_{j 3}$ \\
\hline 1 & 0.0 & -1.0 & & & \\
2 & 1.0 & 0.63212056 & $\underline{0.61269984}$ & & \\
3 & $\underline{0.61209984}$ & 0.07081395 & $\underline{0.56383839}$ & $\underline{0.56744719}$ & \\
4 & $\underline{0.56744719}$ & 0.00047622 & $\underline{0.56714080}$ & $\underline{0.56714312}$ & \\
\hline
\end{tabular}

Figure 5

Rational iteration to a zero of $z-e^{-z}$

4. Convergence Considerations. The results of this section are directed towards an understanding of conditions sufficient for convergence to $z_{0}$ of the columns and diagonals of the $\left\{w_{j k}\right\}$ table, and their asymptotic convergence rates. Specifically, these results are expressible in terms of a function $g(\cdot)$, defined by

$$
g(z)=\frac{z-z_{0}}{f(z)} ; \forall z,
$$

which is assumed to be analytic within a Jordan curve $C$, enclosing the points $\left\{z_{j} ; j=0,1,2, \ldots\right\}$, and bounded on $C$. This amounts to assuming the existence of a neighborhood of $z_{0}$ which excludes all other zeros of $f(\cdot)$, i.e. that $z_{0}$ is an isolated, simple zero of $f(\cdot)$. However, $C$ may contain poles of $f(\cdot)$. 
For any function $h(\cdot)$, let $h\left[z_{1} z_{2} \cdots z_{r}\right]$ denote the $(r-1)$ th divided difference of $h(\cdot)$, based on the abscissae $\left\{z_{j} ; j=1,2, \ldots, r\right\}$.

\section{LEMMA 1.}

$$
z_{0}-w_{j k}=\frac{g\left[z_{j} z_{j+1} \cdots z_{j+k}\right] \Pi_{s=j}^{j+k}\left(z_{0}-z_{s}\right)}{g\left(z_{0}\right)-g\left[z_{0} z_{j} z_{j+1} \cdots z_{j+k}\right] \Pi_{s=j}^{j+k}\left(z_{0}-z_{s}\right)}, \quad \forall \text { relevant } j, k .
$$

Proof. Consider that the function of $z$,

$$
\left(z-w_{1, r-1}\right) g(z)-\left(z-z_{0}\right) Q_{1, r-1}(z),
$$

vanishes, where $z=z_{j} ; j=1,2,3, \ldots, r$, so the polynomial of degree $\leqslant(r-1)$ in $z,\left(z-z_{0}\right) Q_{1, r-1}(z)$, must be the unique polynomial of this degree which agrees at these points, with the function $e(\cdot)$ defined by

$$
e(z)=\left(z-w_{1, r-1}\right) g(z), \quad \forall z .
$$

Hence (e.g. Milne-Thomson [9])

(8) $\left(z-w_{1, r-1}\right) g(z)-\left(z-z_{0}\right) Q_{1, r-1}(z)=e\left[z z_{1} z_{2} \cdots z_{r}\right] \prod_{j=1}^{r}\left(z-z_{j}\right) ; \quad \forall z$, and in particular,

$$
\left(z_{0}-w_{1, r-1}\right) g\left(z_{0}\right)=e\left[z_{0} z_{1} z_{2} \cdots z_{r}\right] \prod_{j=1}^{r}\left(z_{0}-z_{j}\right)
$$

However,

$$
e(z)=\left(z-z_{0}\right) g(z)+\left(z_{0}-w_{1, r-1}\right) g(z)
$$

so, using elementary properties of divided differences, we find

$$
e\left[z_{0} z_{1} z_{2} \cdots z_{r}\right]=g\left[z_{1} z_{2} \cdots z_{r}\right]+\left(z_{0}-w_{1, r-1}\right) g\left[z_{0} z_{1} z_{2} \cdots z_{r}\right],
$$

which yields

$$
z_{0}-w_{1, r-1}=\frac{g\left[z_{1} z_{2} \cdots z_{r}\right] \Pi_{j=1}^{r}\left(z_{0}-z_{j}\right)}{g\left(z_{0}\right)-g\left[z_{0} z_{1} z_{2} \cdots z_{r}\right] \Pi_{j=1}^{r}\left(z_{0}-z_{j}\right)}
$$

when substituted into (9).

By translating this result in the $\left\{w_{j k}\right\}$-table it is clear that the general proposition is also true.

THEOREM 1. If there exists $\gamma$ such that

$$
\frac{\left|z_{0}-z_{j}\right|}{d_{j}} \leqslant \gamma<1 ; \quad d_{j}=\inf _{z \in C}\left|z-z_{j}\right| ; j=0,1,2, \ldots,
$$

then the sequence $\left\{w_{1, r} ; r=1,2,3, \ldots\right\}$ converges to $z_{0}$.

Proof. By a well-known property of divided differences of an analytic function

$$
g\left[z_{1} z_{2} \cdots z_{r}\right]=\frac{1}{2 \pi i} \int_{C} \frac{g(z) d z}{\Pi_{j=1}^{r}\left(z-z_{j}\right)}
$$


and

$$
g\left[z_{0} z_{1} z_{2} \cdots z_{r}\right]=\frac{1}{2 \pi i} \int_{C} \frac{g(z) d z}{\Pi_{j=0}^{r}\left(z-z_{j}\right)} .
$$

Hence, if $L$ is the length of $C$ and $M=\sup _{z \in C}|g(z)|$, we have

$$
\left|g\left[z_{1} z_{2} \cdots z_{r}\right]\right| \leqslant \frac{L M}{2 \pi \Pi_{j=1}^{r} d_{j}}
$$

and

$$
\left|g\left[z_{0} z_{1} z_{2} \cdots z_{r}\right]\right| \leqslant \frac{L M}{2 \pi \Pi_{j=0}^{r} d_{j}} .
$$

Furthermore, $g\left(z_{0}\right) \neq 0$, since $z_{0}$ is a zero of $f(\cdot)$ and $g(\cdot)$ is analytic at $z_{0}$, so from (10) and (12) we see that for any fixed $\epsilon$ satisfying $1>\epsilon>0$, there exists $R$ such that

$$
\frac{\lg \left[z_{0} z_{1} z_{2} \cdots z_{r}\right]\left|\Pi_{j=1}^{r}\right| z_{0}-z_{j} \mid}{\lg \left(z_{0}\right) \mid} \leqslant \epsilon, \quad \forall r>R
$$

Consequently, from Lemma 1,

$$
\begin{aligned}
\left|z_{0}-w_{1, r-1}\right| & \leqslant \frac{\left|g\left[z_{1} z_{2} \cdots z_{r}\right]\right| \Pi_{j=1}^{r}\left|z_{0}-z_{j}\right|}{(1-\epsilon)\left|g\left(z_{0}\right)\right|} \\
& \leqslant \frac{L M \gamma^{r}}{2 \pi(1-\epsilon)\left|g\left(z_{0}\right)\right|}, \quad \forall r>R,
\end{aligned}
$$

from (10) and (11), from which the required result follows.

COROLlary 1. Let $z_{0}^{*}$ be the zero of $f(\cdot)$ nearest to $z_{0}$, and suppose $f(\cdot)$ has only pole-type singularities in the disk $\left\{z:\left|z-z_{0}\right| \leqslant \beta\left|z_{0}^{*}-z_{0}\right|\right\}$, where $0<\beta<1$; then the sequence $\left\{w_{1, r} ; r=1,2,3, \ldots\right\}$ converges to $z_{0}$ if

$$
\frac{\left|z_{j}-z_{0}\right|}{\left|z_{0}^{*}-z_{0}\right|} \leqslant \frac{\beta}{2} ; \quad j=1,2,3, \ldots
$$

Proof. These conditions imply the conditions for Theorem 1, if we take $C$ to be the perimeter of this disk. The result also holds if $z_{0}^{*}$ is any nonpolar singularity of $f(\cdot)$.

COROLlaRY 2. Under the conditions of Theorem 1,

$$
z_{0}-w_{1, r-1} \sim \frac{g\left[z_{1} z_{2} \cdots z_{r}\right] \Pi_{j=1}^{r}\left(z_{0}-z_{j}\right)}{g\left(z_{0}\right)},
$$

for large $r$. 
Proof. Observe that $g\left(z_{0}\right) \neq 0$ and from (12)

$$
\begin{aligned}
& \left|g\left[z_{0} z_{1} z_{2} \cdots z_{r}\right]\right| \prod_{j=1}^{r}\left|z_{0}-z_{r}\right| \\
& \quad \leqslant \frac{L M}{2 \pi d_{0}} \prod_{j=1}^{r}\left|\frac{z_{0}-z_{j}}{d_{j}}\right| \leqslant\left|g\left(z_{0}\right)\right|\left[\frac{L M}{2 \pi d_{0} \cdot\left|g\left(z_{0}\right)\right|}\right] \gamma^{r} \rightarrow 0 \quad \text { as } r \rightarrow \infty
\end{aligned}
$$

so the result follows from Lemma 1 .

Corollary 3. If the sequence $\left\{z_{j} ; j=1,2,3, \ldots\right\}$ converges to $z_{0}$, then, for fixed $k$ and large $j$,

$$
z_{0}-w_{j k} \sim \frac{g^{(k)}\left(z_{0}\right)}{k ! g\left(z_{0}\right)} \prod_{s=j}^{j+k}\left(z_{0}-z_{s}\right) .
$$

Proof. This result is obvious from Lemma 1 and elementary properties of divided differences.

COROLlaRY 4. If the sequence $\left\{z_{j} ; j=1,2,3, \ldots\right\}$ converges to $z_{0}$, then, for every fixed $k \geqslant 1$,

$$
w_{j k} \rightarrow z_{0} \text { as } j \rightarrow \infty .
$$

The above results are largely independent of the rule for augmenting the sequence $\left\{z_{j} ; j=1,2, \ldots, r\right\}$. Theorems 2 and 4 , below, effectively confirm convergence under simple augmentation rules, if $z_{1}$ and $z_{2}$ are chosen close enough to $z_{0}$ in comparison to the distance between $z_{0}$ and $z_{0}^{*}$.

Using the same notation and region of analyticity as in Corollary 1 , we choose a positive quantity $\gamma$, subject to the condition

$$
0<\gamma(1+2 \gamma)<\frac{\left|g\left(z_{0}\right)\right|}{M} \leqslant 1 ;
$$

this is always possible, the second inequality deriving directly from the Maximum Modulus Principle. From (13) it is easy to verify that

$$
\begin{gathered}
0<\gamma<\frac{\left|g\left(z_{0}\right)\right|}{M}, \text { and } \\
0<\gamma<\frac{\sqrt{5}-1}{4} \simeq 0.3090 .
\end{gathered}
$$

THEOREM 2. If

$$
\left|\frac{z_{0}-z_{j}}{d_{j}}\right| \leqslant \gamma ; \quad j=1,2,
$$

and the sequence $\left\{z_{j} ; j=1,2,3, \ldots\right\}$ is augmented by means of the rule

$$
z_{r+1}=w_{1, r-1} ; \quad r=2,3,4, \ldots,
$$

then $z_{j} \rightarrow z_{0}$ as $j \rightarrow \infty$. 
Proof. We first show by induction that $(16)$ is true for all $j \geqslant 0$. Hypothesizing that $(16)$ is true for $1 \leqslant j \leqslant r$, we define

$$
\mu=\frac{M}{\left|g\left(z_{0}\right)\right|}\left|\prod_{j=1}^{r}\right| \frac{z_{0}-z_{j}}{d_{j}} \mid
$$

and observe from (12) that

$$
\left|g\left(z_{0}\right)\right|^{-1}\left|g\left[z_{0} z_{1} z_{2} \cdots z_{r}\right]\right| \prod_{j=1}^{r}\left|z_{0}-z_{j}\right| \leqslant \frac{M L}{2 \pi d_{0}\left|g\left(z_{0}\right)\right|} \prod_{j=1}^{r}\left|\frac{z_{0}-z_{j}}{d_{j}}\right|
$$

However,

$$
L=2 \pi d_{0} \text {, }
$$

since $C$ is a circle centered on $z_{0}$, so, using (13), (14) and (16),

(19) $\left|g\left(z_{0}\right)\right|^{-1}\left|g\left[z_{0} z_{1} z_{2} \cdots z_{r}\right]\right| \prod_{j=1}^{r}\left|z_{0}-z_{j}\right| \leqslant \mu \leqslant \frac{M \gamma^{r}}{\left|g\left(z_{0}\right)\right|} \leqslant \frac{M \gamma^{2}}{\left|g\left(z_{0}\right)\right|}<\frac{\left|g\left(z_{0}\right)\right|}{M}$

for all $r \geqslant 2$. Notice also, from (13) and (19), that

$$
\mu \gamma(1+2 \gamma)<\gamma^{2}, \text { i.e. } \mu<\frac{\gamma}{1+2 \gamma}<\gamma, \quad \forall r>1 .
$$

Thus, from Lemma 1, we have

$$
\begin{aligned}
\left|z_{0}-z_{r+1}\right|=\left|z_{0}-w_{1, r-1}\right| & \leqslant \frac{\left|g\left[z_{1} z_{2} \cdots z_{r}\right]\right| \Pi_{j=1}^{r}\left|z_{0}-z_{j}\right|}{\left|g\left(z_{0}\right)\right|-\left|g\left[z_{0} z_{1} z_{2} \cdots z_{r}\right]\right| \Pi_{j=1}^{r}\left|z_{0}-z_{j}\right|} \\
& \leqslant \frac{d_{0} M \Pi_{j=1}^{r}\left|\left(z_{0}-z_{j}\right)\right| d_{j} \mid}{\left|g\left(z_{0}\right)\right|-M \Pi_{j=1}^{r}\left|\left(z_{0}-z_{j}\right) / d_{j}\right|},
\end{aligned}
$$

making use of (11), (12), (18) and the fact that (20) implies that the denominator is positive. Hence

$$
\left|z_{0}-z_{r+1}\right| \leqslant \frac{d_{0} \mu}{1-\mu}=\left(d_{r+1}+\left|z_{0}-z_{r+1}\right|\right) \frac{\mu}{1-\mu},
$$

i.e.

$$
\left|\frac{z_{0}-z_{r+1}}{d_{r+1}}\right| \leqslant \frac{\mu}{1-2 \mu}<\frac{\gamma /(1+2 \gamma)}{1-2 \gamma /(1+2 \gamma)}=\gamma
$$

making use of (15) and (20). Since the hypothesis is assumed true for $r=2$, in the conditions of the proposition, the induction is complete. The conditions for the validity of Theorem 1 are thus satisfied, so the proposition is proved.

COROLlaRY 5. If the sequence $\left\{z_{j} ; j=1,2,3, \ldots\right\}$ converges to $z_{0}$, under the augmentation rule of Theorem 2 , and $z_{0} \neq z_{j} ; j=1,2,3, \ldots$, then, presuming the limit exists,

$$
\lim _{r \rightarrow \infty} \frac{\left|z_{0}-z_{r+1}\right|}{\left|z_{0}-z_{r}\right|^{2}}=\frac{1}{\left|z_{0}^{*}-z_{0}\right|}
$$


Proof. From Corollary 2 and the augmentation rule we have

$$
z_{0}-z_{r+1} \sim \frac{g\left[z_{1} z_{2} \cdots z_{r}\right]}{g\left(z_{0}\right)} \prod_{j=1}^{r}\left(z_{0}-z_{j}\right)
$$

and

$$
z_{0}-z_{r} \sim \frac{g\left[z_{1} z_{2} \cdots z_{r-1}\right]}{g\left(z_{0}\right)} \prod_{j=1}^{r-1}\left(z_{0}-z_{j}\right), \quad \text { for large } r
$$

Thus

$$
\frac{z_{0}-z_{r+1}}{\left(z_{0}-z_{r}\right)^{2}} \sim \frac{g\left[z_{1} z_{2} \cdots z_{r}\right]}{g\left[z_{1} z_{2} \cdots z_{r-1}\right]}
$$

and it may be shown (Larkin [6]) that

$$
\left|\frac{g\left[z_{1} z_{2} \cdots z_{r}\right]}{g\left[z_{1} z_{2} \cdots z_{r-1}\right]}\right| \rightarrow \frac{1}{\left|z_{0}^{*}-z_{0}\right|} \text { as } r \rightarrow \infty,
$$

whenever the limit exists.

Remark 1. The asymptotic order of convergence of the process is 2 , and so are its efficiency index and informational efficiency. This asymptotic behavior is identical with that of the method of polynomial inverse interpolation (e.g. [7]).

Numerical Example. To illustrate that this asymptotic pattern can become fairly quickly established we choose

$$
f(z)=z(1+z) ; \quad \forall z,
$$

and iterate towards the root $z_{0}=0$. Obviously $z_{0}^{*}=-1$ so $\left|z_{0}^{*}-z_{0}\right|=1$. Starting with $z_{1}=0.5, z_{2}=0.4$, relevant quantities are shown in Figure 6 .

\begin{tabular}{|l|l|c|}
\hline$j$ & \multicolumn{1}{|c|}{$z_{j}$} & $\left|z-z_{j+1}\right| /\left|z_{o}{ }^{-z_{j}}\right|^{2}$ \\
\hline 1 & 0.5 & \\
2 & 0.4 & 0.5691 \\
3 & 0.10526316 & 0.8261 \\
4 & $9.153318 .10^{-3}$ & 0.9820 \\
5 & $8.227702 .10^{-5}$ & 0.9998 \\
6 & $6.768394 .10^{-9}$ & \\
\hline
\end{tabular}

FIGURE 6

Asymptotic behavior of the method

If there is more than one "nearest neighbor root" to $z_{0}$ the pattern is more complicated, but the behavior expressed by Corollary 2 may still be discernible. 
THEOREM 3. If the augmentation rule of Theorem 2 is replaced by the rule

$$
z_{r+1}= \begin{cases}w_{1, r-1} ; & r=2,3, \ldots, k+1, \\ w_{r-k, k} ; & r=k+2, k+3, \ldots,\end{cases}
$$

the sequence $\left\{z_{j} ; j=1,2,3, \ldots\right\}$ still converges to $z_{0}$.

Proof. From the analysis of Theorem 2 we know that

$$
\left|\frac{z_{0}-z_{j}}{d_{j}}\right| \leqslant \gamma ; \quad j=1,2, \ldots, k+2 .
$$

We now show that the sequence $\left\{\left|z_{0}-z_{j}\right| ; j=k+3, k+4, \ldots\right\}$ is bounded by a geometrically decreasing sequence, again using an inductive argument.

Hypothesize that $(21)$ is also true for $\{j=k+3, k+4, \ldots, r\}$ then, from Lemma 1 , we have

$$
\frac{z_{0}-z_{r+1}}{z_{0}-z_{r}}=\frac{g\left[z_{r-k} z_{r-k+1} \cdots z_{r}\right] \Pi_{j=r-k}^{r-1}\left(z_{0}-z_{j}\right)}{g\left(z_{0}\right)-g\left[z_{0} z_{r-k} z_{r-k+1} \cdots z_{r}\right] \Pi_{j=r-k}^{r}\left(z_{0}-z_{j}\right)}, \quad \forall r \geqslant k+1,
$$

so, by arguments similar to those used in Theorem 1,

$$
\left|\frac{z_{0}-z_{r+1}}{z_{0}-z_{r}}\right| \leqslant \frac{\left(M d_{0} / d_{r}\right) \Pi_{j=r-k}^{r-1}\left|\left(z_{0}-z_{j}\right) / d_{j}\right|}{\left|g\left(z_{0}\right)\right|-M \Pi_{j=r-k}^{r}\left|\left(z_{0}-z_{j}\right) / d_{j}\right|} .
$$

However, $d_{0}=\left|z_{0}-z_{r}\right|+d_{r}$ so relation (22) can be expressed as

$$
\begin{aligned}
\left|\frac{z_{0}-z_{r+1}}{z_{0}-z_{r}}\right| & \leqslant \frac{\left[1+\left|z_{0}-z_{r}\right| / d_{r}\right] M /\left|g\left(z_{0}\right)\right| \Pi_{j=r-k}^{r-1}\left|\left(z_{0}-z_{j}\right) / d_{j}\right|}{1-M /\left|g\left(z_{0}\right)\right| \Pi_{j=r-k}^{r}\left|\left(z_{0}-z_{j}\right) / d_{j}\right|} \\
& \leqslant \frac{(1+\gamma) \gamma^{k} M / \lg \left(z_{0}\right) \mid}{1-\gamma^{k+1} M / \lg \left(z_{0}\right) \mid} \leqslant \frac{(1+\gamma) \gamma^{k-1} /(1+2 \gamma)}{1-\gamma^{k} /(1+2 \gamma)}
\end{aligned}
$$

from (13); i.e.

$$
\left|\frac{z_{0}-z_{r+1}}{z_{0}-z_{r}}\right| \leqslant \frac{(1+\gamma) \gamma^{k-1} /(1+2 \gamma)}{1-\gamma /(1+2 \gamma)}=\gamma^{k-1}<\gamma<1 .
$$

Thus (21) will be true for $j=r+1$, and the induction is valid; also (23) confirms that the sequence $\left\{\left|z_{0}-z_{j}\right| ; j=k+2, k+3, \ldots\right\}$ is indeed bounded by a geometrically decreasing sequence, so the proposition is proved.

COROLlaRY 6. If the sequence $z_{j} ; j=1,2,3, \ldots$, converges to $z_{0}$ under the augmentation rule of Theorem 3 , and $z_{0} \neq z_{j} ; j=1,2,3, \ldots$, then

$$
\lim _{r \rightarrow \infty} \frac{\left|z_{0}-z_{r+1}\right|}{\left|z_{0}-z_{r}\right|^{p}}=\left|\frac{g^{(k)}\left(z_{0}\right)}{k ! g\left(z_{0}\right)}\right|^{(p-1) / k},
$$

where $p$ is the unique positive root of the equation

$$
p^{k+1}=\sum_{j=0}^{k} p^{k}
$$


Proof. From Corollary 3 and the augmentation rule we have

$$
z_{0}-z_{r+1} \sim \frac{g^{(k)}(0)}{k ! g\left(z_{0}\right)} \prod_{j=r-k}^{r}\left(z_{0}-z_{j}\right)
$$

After taking absolute values and then logarithms on both sides of (25), thus forming an approximate linear recurrence relation, standard arguments (e.g. Traub [14]) lead to the required result.

Remark 2. It is, of course, well known that $p$ increases monotonically from $(\sqrt{5}-1) / 2$ to 2 as $k$ increases from 2 to $\infty$ (e.g. Traub [14]).

Corollary 7.

$$
\varlimsup_{k \rightarrow \infty}\left|\frac{g^{(k)}\left(z_{0}\right)}{k ! g\left(z_{0}\right)}\right|^{(p-1) / k}=\frac{1}{\left|z_{0}^{*}-z_{0}\right|} .
$$

Proof. On summing the geometric series in (24), we easily find that

$$
p-1=1-\frac{1}{p^{k+1}}
$$

so

$$
\left|\frac{g^{(k)}\left(z_{0}\right)}{k ! g\left(z_{0}\right)}\right|^{(p-1) / k}=\left[\left|\frac{g^{(k)}\left(z_{0}\right)}{k !}\right|^{1 / k}\left|\frac{1}{g\left(z_{0}\right)}\right|^{1 / k}\right]^{1-1 / p^{k+1}}
$$

However, for large enough $k$ the effect of the power $1 / p^{k+1}$ becomes negligible,

$$
\lim _{k \rightarrow \infty}\left|\frac{1}{g\left(z_{0}\right)}\right|^{1 / k}=1
$$

and, by the Cauchy-Hadamard Theorem,

$$
\varlimsup_{k \rightarrow \infty}\left|\frac{g^{(k)}\left(z_{0}\right)}{k !}\right|^{1 / k}=\frac{1}{\left|z_{0}^{*}-z_{0}\right|},
$$

so the proposition is proved.

Department of Computing and Information Science

Queen's University

Kingston, Ontario, Canada

1. A. C. AITKEN, "On interpolation by proportional parts, without the use of differences," Proc. Roy. Soc. Edinburgh, v. 53, 1932, pp. 54-78.

2. P. JARRATT \& D. NUDDS, "The use of rational functions in the iterative solution of equations on a digital computer," Comput. J., v. 8, 1965, pp. 62-65.

3. P. JARRATT, "A note on the asymptotic error constant of a certain method for solving equations," Comput. J., v. 9, 1967, pp. 408-409.

4. P. JARRATT, "A review of methods for solving nonlinear equations in one variable," in Numerical Methods for Non-Linear Algebraic Equations (P. Rabinowitz, Ed.), Gordon and Breach, London, 1970.

5. F. M. LARKIN, "Some techniques for rational interpolation," Comput. J., v. 10, 1967, pp. $178-187$.

6. F. M. LARKIN, The Newton Series in Analytic Function Theory, Tech. Report 79-72, Dept. of Computing and Information Science, Queen's University, Kingston, Ontario, 1979. 
7. F. M. LARKIN, On a Generalization of Aitken's $\delta^{2}$-Process, Tech. Report 79-74, Dept. of Computing and Information Science, Queen's University, Kingston, Ontario, 1979.

8. F. M. LARKIN, On the Acceleration of Certain Sequences by Rational Interpolation, Tech. Report 79-75, Dept. of Computing and Information Science, Queen's University, Kingston, Ontario, 1979.

9. L. M. MILNE-THOMSON, The Calculus of Finite Differences, Macmillan, London, 1965.

10. E. H. NEVILLE, "Iterative interpolation," J. Indian Math. Soc., v. 20, 1936, pp. 87120.

11. A. M. OSTROWSKI, Solution of Equations and Systems of Equations, Academic Press, New York, 1966.

12. J. STOER, “Algorithmen zur Interpolation mit rationalen Funktionen," Numer. Math., v. 3, 1961, pp. 285-304.

13. L. TORNHEIM, "Convergence of multipoint iterative methods," J. $A C M$, v. 11, 1964, pp. 210-220.

14. J. F. TRAUB, Iterative Methods for the Solution of Equations, Prentice-Hall, Englewood Cliffs, N. J., 1964.

15. P. WYNN, "On a procrustean technique for the numerical transformation of slowly convergent sequences and series," Proc. Cambridge Philos. Soc., v. 52, 1958, pp. 663-671.

16. P. WYNN, "Über einen Interpolations Algorithmus and gewisse andere Formeln, die in der Theorie der Interpolation durch rationale Functionen bestehen," Numer. Math., v. 2, 1960, pp. 151-182. 